of high-performance applications, connections and protocols that would transform the public Internet into a high-speed network. This marks a return to a more interventionist approach to Internet development in the United States.

The privatization of NSFnet led to a slip in Internet development, Strawn concedes. There is now a growing realization that the state has a role to play, he says. "People have migrated towards the view that networking advances could be accelerated by reinvigoration of the public-private partnership that brought us the Internet in the first place."

\section{Major benefits for researchers}

The United States does not intend to create private research intranets closed off from the public Internet, as still happens in Europe and Japan. Although the vBNS is for the moment a private intranet, it will provide the research community with high levels of performance until these can be delivered by the public Internet, says Strawn. "There is no intention of setting up a separate infrastructure [in the long term]."

The costs of maintaining intranets are exorbitant. Many European research organizations now believe the best performance at lowest cost will eventually come from the public Internet, which will quickly evolve to high speeds while delivering economies of scale (see Nature 378, 380; 1996).

Scott Bradner, a senior technical consultant at Harvard University, agrees, pointing out that researchers have not needed to build an independent telephone network. "There is no long-term viable solution which excludes the public Internet," he says.

Major benefits to scientists will come from a new set of protocols being tested in second-generation Internet projects. These will enable users to select the level of performance they want, with a sliding scale of charges replacing the system where most users pay a flat monthly connection fee.

The idea of prioritizing traffic in this way was until recently opposed by many Internet users attached to the principle of flat-fee access. But it is now gaining widespread acceptance as users grow to realize that popular video applications, like many research applications, will require guaranteed levels of performance if they are to work properly.

The new protocols may also ease the current political problem of sharing the high costs of international links. They would allow countries to be guaranteed a specific share of the bandwidth corresponding to the amount of its subscription, which is impossible at present. Bottlenecks on transcontinental links are a major problem.

\title{
'Europe must do more to promote networks'
}

[PARIS] The European Union

(EU) should play a much greater role in promoting both electronic networks for researchers and the public Internet, says a report by one of the European

Commission's advisory groups. "This is a plea for recognition [of the

importance of the Internet] at the highest levels of the union," says the report. "Europe has to wake up to the Internet at many levels."

The group's members are communications experts from universities, government research organizations and private industry. It was set up last year in response to the creation of several secondgeneration Internet initiatives in the United States. Its report, The Future of the Internet - What Role for Europe?, says there is a need for "urgent and effective action, and appropriate budgetary commitments" to catch up with the United States.
The commission also needs a dedicated body to deal with the Internet, according to the report, which points out that at present "each major Internet development made outside Europe has to be analysed and reacted to by fairly ad hoc groups of EU staff and outside experts". The group says it is "urgent" that the commission should appoint experts to track the progress of the US initiatives, which it argues represent a significant development.

The report says that "excessive line costs are still the biggest barrier to the spread of the Internet in Europe". It calls on the EU to act quickly to ensure that the liberalization of European telecommunications companies scheduled for next year results in a fall in bandwidth costs. This will be critical if Europe is to move towards the high-speed networks envisaged in the US second-generation
Internet project, says the report. High speeds are needed by highly demanding applications.

It is "essential" that the EU makes funding available for a follow-up to the Ten-34 project to bring links between national research networks up to 155 and 622 Mbps, the report says. It also recommends that European countries build shared regional infrastructure, along the lines of the gigapops planned in the US Internet-2 initiative (see above).

The wider thrust of the report is that the EU should play a greater role in supporting Intemet research and development and infrastructure more generally. Areas where the union could help include supporting the development of Internet protocols for prioritizing traffic and encouraging the deployment of alternative technologies for home access to the Internet, such as cable television and satellite.
Concern about global connectivity recently prompted the International Committee for Future Accelerators - which promotes collaboration in the building and use of accelerators - to set up an ad hoc panel to address the issue. "This is the first time that ICFA have recognized [Internet connectivity] as a serious issue," says David Williams, chair of the panel and former head of the computing and networks division of the European Laboratory for Particle Physics.

Progress in establishing better international links has been hampered in particular by the political difficulties of negotiating them. European research organizations, for example, resent paying 90 per cent of the costs of the shared links to the United States (with NSF paying the rest), whereas each accounts for about the same amount of traffic over the link.

Some of this imbalance is because the cost of bandwidth in European countries where telecommunications companies still have monopolies is an order of magnitude higher than in the United States. Indeed, the high cost of bandwidth remains the major obstacle to general development of the Internet in Europe, although many believe that the scheduled deregulation of telecommunications companies within the union next year will bring about major price reductions.

But Dai Davies, general manager of Dante, attributes much of the imbalance to Europe's relatively weak negotiating position in the past. "Europe does not have a single voice on this, and the Americans have been able to divide and conquer." He says there is also an "asymmetry" in that all European countries have state-supported research intranets whereas Internet services in the United States are mainly supplied by commercial providers which are mainly concerned with local connectivity and "don't care about research links to Europe”.

\section{Competition sparks alliances}

France, Germany, Italy, the United Kingdom and the European Commission have adopted a common position for this month's meeting on the proposed G7 Global Interconnection of Broadband Networks project. Europe will for the first time have a single voice on the issue, says Davies. He predicts that the Americans "are going to get pinned down" for not contributing sufficiently on global connectivity.

Poor international and regional connections have also resulted from the reluctance of telecommunications operators to link up with each others' networks. Global competition is now prompting a spate of joint ventures - such as Global One, a joint network between France Telecom, Deutsche Telecom and Sprint - which will bring faster and more reliable connections. Mergers and alliances will be extremely important for connectivity", says Strawn. 